\title{
ANOTHER NOTE ON KEMPISTY'S GENERALIZED CONTINUITY
}

\author{
J.P. LEE \\ Department of Mathematics \\ State University of New York \\ College at 01d Westbury \\ O1d Westbury, NY 11568 \\ and

\section{Z. PIOTROWSKI} \\ Department of Mathematical \& Computer Sciences \\ Youngstown State University \\ Youngstown, $\mathrm{OH} 44555$ \\ (Received September 23, 1987 and in revised form November 11, 1987) \\ ABSTRACT. Under a fairly mild completeness condition on spaces $Y$ and $Z$ we show that \\ every $\mathrm{x}$-continuous function $\mathrm{f}: \mathrm{X} \times \mathrm{Y} \times \mathrm{Z} \rightarrow \mathrm{M}$ has a "substantial" set $\mathrm{C}(\mathrm{f})$ of points \\ of continuity. Some odds and ends concerning a related earlier result shown by the \\ authors are presented. Further, a generalization of S. Kempisty's ideas of \\ generalized continuity on products of finftely many spaces is offered. As a \\ corollary from the above results, a partial answer to M. Talagrand's problem is
} provided.

KEYS WORDS AND PHRASES. quasi-continuity, $x$-continuity, separate and joint continuity, Baire spaces.

1980 AMS SUBJECT CLASSIFICATION CODE. 54C10, 54C30.

1. $\mathrm{x}$-CONTINUITY.

The notion of symmetric quasi-continuity introduced by $S$. Kempisty [1] has been generalized in Lee and Piotrowski [2], to $\mathrm{x}$-continuity. In what follows let $\mathrm{X}, \mathrm{Y}, \mathrm{Z}$ and $T$ be spaces. Following Lee and Piotrowski [2] a function $f: X \times Y \times Z \rightarrow T$ is $x$-continuous if for every $(p, q, r) \varepsilon X \times Y \times Z$, for every neighborhood $U \times V \times W$ of $(p, q, r)$ and for every neighborhood $N$ of $f(p, q, r)$ there exists a neighborhood $U^{\prime}$ of $p$ with $U^{\prime} \subset U$ and nonempty open sets $V^{\prime}$ and $W^{\prime}$ with $V^{\prime} \subset V$ and $W^{\prime} \subset W$ such that for all $(x, y, z) \varepsilon U^{\prime} \times V^{\prime} \times W^{\prime}$ it follows that $f(x, y, z) \varepsilon N$.

We shall first show that under certain general assumptions concerning the spaces, $x$-continuous functions have "large" sets of points of joint continuity. In order to do this we first list some necessary definitions.

Let $A$ be an open covering of a space $X$. Then a subset $S$ of $X$ is said to be A-small if $S$ is contained in a member of $A$. A space $X$ is called strongly countably complete if there exists a sequence $\left\{A_{1}: i=1,2, \ldots\right\}$ of open coverings of $x$ such that and sequence $\left\{F_{i}\right\}$ of $A_{i}$-small, closed subsets of $X$ for which $F_{i} \supset F_{i+1}$ has a non- 
empty intersection.

The class of strongly countably complete spaces include countably compact and complete metric spaces. This fact follows easily from a theorem due to A. Arhandel'skii [3] and Z. Frolík [4] which states that in the class of completely regular spaces, Cech-complete and strongly countably complete spaces coincide (Engelking [5]), see also Frolík [4], where some other properties of these spaces such as their invariance under taking closed, open subspaces or products are discussed.

A space $X$ is called quasi-regular, (Oxtoby [6]) if for every nonempty open set $u$, there is a nonempty open set $V$ such that $c 1 v \subset u$. Obviously, every regular space is quasi-regular.

Let us recall that a function $f: X \times Y \rightarrow Z$ is said to be quasi-continuous with respect to $x$, (Kempisty [1] p.188,) if for every $(p, q) \varepsilon X \times Y$, fore very neighborhood $N$ of $f(p, q)$ and every neighborhood $U \times V$ of $(p, q)$ there exists a neighborhood $U^{\prime}$ of $p$ with $U^{\prime} \subset U$ and a nonempty open set $V^{\prime} \subset V$ such that for $a 11(x, y) \varepsilon U^{\prime} \times V^{\prime}$ we have $f(x, y) \in N$. Quasi-continuity with respect to y can be defined similarly.

LEMMA 1. (Lee and Piotrowski [2], Lemma 3 p. 383). Let X, Y, Z and T be spaces and let $\mathrm{F}: \mathrm{X} \times \mathrm{Y} \times \mathrm{Z} \rightarrow \mathrm{T}$ be a function. Then $\mathrm{f}$ is $\mathrm{x}$-continuous if and only if $\mathrm{g}: \mathrm{X} \times \mathrm{S} \rightarrow \mathrm{T}$ is quasi-continuous with respect to $\mathrm{x}$, where $\mathrm{S}=\mathrm{Y} \times \mathrm{Z}$ and $g(x,(y, z))=f(x, y, z)$.

THEOREM 2. Let $X$ be a space, $Y$ and $Z$ be spaces such that $Y \times Z$ is quasi-regular, strongly countably complete and let $M$ be metric. If $f: X \times Y \times Z \rightarrow M$ is $X$-continuous, then for every $x \in X$, the set $C(f)$ of continuity points of $f$ is dense $G_{\delta}$ subset in $\{\mathrm{X}\} \times \mathrm{Y} \times \mathrm{Z}$.

PROOF. In view of Lemma 1 it is sufficient to prove the following:

CLAIM. Let $X$ be a space, $Y$ be a quasi-regular, strongly countably complete and $\mathrm{Z}$ be metric. If $\mathrm{f}: \mathrm{X} \times \mathrm{Y} \rightarrow \mathrm{Z}$ is quasi-continuous with respect to $\mathrm{x}$, then for all $X \varepsilon X$ the set of points of joint continuity of $f$ is a dense $G_{\delta}$ subset of $\{x\} \times Y$.

PROOF. First we will prove that the set of points of joint continuity of $f$ is dense in $\{x\} \times Y$. Let $x \in X, y \in Y$ and $U \times V$ be any neighborhood $U$ of $x$, contained in $U$, and a nonempty open set $V^{1} \subset V$ such that for a11 ( $\left.x^{\prime}, y^{\prime}\right)$ and ( $\left.x^{\prime \prime}, y^{\prime \prime}\right)$ in $U^{l} \times V^{l}$, we have $\rho\left(f\left(x^{\prime}, y^{\prime}\right), f\left(x^{\prime \prime}, y^{\prime \prime}\right)\right)<1$. Without loss of generality we may assume that $V^{1}$ is contained in an element $A_{1}$ of the covering $A_{1}$ of $Y$. Let $W^{1}$ be a nonempty open set such that $c 1 W^{1} \subset V^{1}$. So $c l W^{1}$ is $A_{1}$-small. Then $U^{1} \times W^{1}$ is a neighborhood of $\left(x, y_{1}\right)$, where $u_{1} \varepsilon W^{1}$, and since $f$ is quasi-continuous with respect to $x$ at $\left(x, y_{1}\right)$, there is a neighborhood $U^{2}$ of $x$, contained in $U^{l}$ and a nonempty open set $V^{2} \subset W^{1}$, such that for $a l l\left(x^{\prime}, y^{\prime}\right)$ and $\left(x^{\prime \prime}, y^{\prime \prime}\right)$ in $U^{2} \times V^{2}$ we have $\rho\left(f\left(x^{\prime}, y^{\prime}\right)\right.$, $\left.f\left(x^{\prime \prime}, y^{\prime \prime}\right)\right)<\frac{1}{2}$. Similarly, we may assume that $V^{2}$ is contained in an element $A_{2}$ of the covering $A_{2}$. Let $W^{2}$ be a nonempty open set such that $c 1 W^{2} \subset V^{2}$. We see, that c1 $W^{2}$ is $A_{2}$-small.

Now, proceeding by induction we get a neighborhood $U^{n} \times v^{n}$ of $\left(x, y_{n}\right), y_{n} \varepsilon v^{n}$, such that for all $\left(x^{\prime}, y^{\prime}\right)$ and $\left(x^{\prime \prime}, y^{\prime \prime}\right)$ in $U^{n} \times v^{n}$, we have $\rho\left(f\left(x^{\prime}, y^{\prime}\right), f\left(x^{\prime \prime}, y^{\prime \prime}\right)\right)<\frac{1}{n}$ and that $v^{n}$ is contained in an element $A_{n}$ of the covering $A_{n}$ of $Y$. Moreover, there is a nonempty open sets $W^{n}$ such that $v^{n+1} \subset c 1 W^{n} \subset v^{n}$. Thus each cl $W^{n}$ is $A_{n}-\operatorname{smal1}$, obviously $c 1 W^{n} \supset c 1 W^{n+1}$. Since $Y$ is strongly countably complete $\bigcap_{n=1}^{\infty} c 1 W^{n} \neq \emptyset$. Let 
$y^{*} \varepsilon \prod_{n=1}^{\infty} c 1 w^{n} . \quad$ Then

$$
\left(x, y^{*}\right) \varepsilon \prod_{n=1}^{\infty}\left(U^{n} \times c 1 W^{n}\right) \subset \prod_{n=1}^{\infty}\left(U^{n} \times v^{n}\right) \subset U \times v .
$$

Thus $\left(\mathrm{x}, \mathrm{y}^{*}\right) \varepsilon(U \times \mathrm{V}) \cap(\{\mathrm{x}\} \times \mathrm{y})$ and $\left(\mathrm{x}, \mathrm{y}^{*}\right)$ is a point of joint continuity of $f$. This shows the density of the set of points of joint continuity of $f$ in the set $\{\mathrm{X}\} \times \mathrm{Y}$.

The proof that this set is $G_{\delta}$ subset of $\{x\} \times Y$ easily follows, when we recall that the function $f$ takes values in the metric space $Z$. This completes the proof of Claim.

Thus, Theorem 2 is shown.

The forthcoming, Proposition 3 is contained in Lemma 5.1 of [6], since any quasi-regular strongly countably complete space is pseudo-complete; take $B(n)=$ the class of all nonempty open sets that are $A_{n}-\operatorname{sma} 11$. Then $\{B(n)\}$ is a sequence of (pseudo-) bases that shows $X$ to be pseudo-complete.) We would like to thank the referee who make the above observation.

PROPOSITION 3. (Oxtoby [6], Lemma 5.1) Every quasi-regular strongly countably complete space $X$ is a Baire space.

REMARK 4. Observe that neither base countability nor metrizability assumptions are made on the considered spaces X, Y, Z in Theorem 1 while in Theorem 2 of [2] the same conclusion concerning the set of points of continuity is obtained under an extra assumption that $X$ is first countable, $Y$ is Baire, $Z$ is second countable in a neighborhood of any of its points and such that $Y Z$ is Baire.

2. CONDITIONS IMPLYING $x$-CONTINUITY - COUNTER-EXAMPLES.

Given spaces $\mathrm{X}$ and $\mathrm{Y}$; a function $\mathrm{f}: \mathrm{X} \rightarrow \mathrm{Y}$ is said to be quasi-continuous (Martin [8], compare Kempisty [1]) if for every $x \in X$ and for every neighborhood $U$ of $x$ and for every neighborhood $V$ of $f(x)$ have: $U \cap$ Int $f(V) \neq \emptyset$.

The main result of Lee and Piotrowsk [2] is the following:

THEOREM A. (Lee and Plotrowski [2], Theorem 1, p. 383). Let X be first countable, $Y$ be Baire, $Z$ be second countable such that $Y \times Z$ is Baire and let $T$ be regular. If $\mathrm{f}: \mathrm{X} \times \mathrm{Y} \times \mathrm{Z} \rightarrow \mathrm{T}$ is:

(1) continuous at $X \times\{y\} \times\{z\}, y \in Y, z \varepsilon Z$, and

(2) quasi-continuous at points of $\{\mathrm{X}\} \times \mathrm{Y} \times\{\mathrm{z}\}$ for all $\mathrm{X} \varepsilon \mathrm{X}$ and $\mathrm{z} \varepsilon \mathrm{Z}$, and

(3) quasi-continuous at points of $\{x\} \times\{y\} \times Z$ for all $x \in X$ and $y \varepsilon Y$

then $f$ is $x$-continuous.

The first natural question which comes up is to check whether the converse of Theorem A is true. Apparently, the following Example 5 settles this question in the negative.

EXAMPLE 5. Let $\mathrm{f}: \mathbb{R}^{3} \rightarrow \mathbb{R}$ be defined by

$$
f(x, y, z)=\begin{aligned}
& \sin \frac{1}{x^{2}+y^{2}+z^{2}} \\
& 0, \text { otherwise }
\end{aligned} \text { if }(x, y, z) \neq(0,0,0)
$$


The function $f$ is $x$-continuous, however, fixing $y=0=z$ we obtain that $f(x, 0,0)$ is not continuous.

Now we shall investigate the necessity of the assumptions in Theorem $\mathrm{A}$, in particular:

(*) - continuity of $f$ at points of $x \times\{y\} \times\{z\}$

(**) - quasi-continuity of $f$ at points of $\{x\} \times Y \times\{z\}$, and

$(* * *)$ - quasi-continuity of $f$ at points of $\{x\} \times\{y\} \times z$.

In what follows (Examples 6 and 7) such constructions will be provided.

EXAMPLE 6. The assumption $(*)$ is essential. In fact, let us consider a function $f:[-1,1]^{3} \rightarrow \mathbb{R}^{3}$ given as follows

$$
f(x, y, z)=\begin{aligned}
& (x, y, z+1), \text { if }(x, y, z) \varepsilon[0,1] \times[0,1] \times[0,1] \\
& (x, y, z-1), \text { if }(x, y, z) \varepsilon[-1,0] \times[-1,0] \times[-1,0] \\
& (x, y, z), \text { otherwise }
\end{aligned}
$$

A standard verification that $f$ has the required property, (namely $f$ is not $x$-continuous at $(0,0,0)$ ) is left to the reader. Using somewhat more complex, but still elementary techniques we shall show that also ( $* *$ ) (as well as ( $* * *)$ ) is essential. In fact, we have

EXAMPLE 7. Consider the function $g:[-1,1]^{3} \rightarrow \mathbb{R}^{3}$ given as follows:

$$
\begin{aligned}
g(x, y, z)= & (x, y, z+1) \text { if }(x, y, z) \varepsilon[-1,1] \times\left[-\frac{1}{2}, 1\right] \times x \\
& \times\left\{\left(\left[-\frac{1}{2}, \frac{1}{2}\right) \cap I Q\right) U\left[\frac{1}{2}, 1\right]\right\} \\
& (x, y, z), \text { otherwise }
\end{aligned}
$$

Again, we leave to the interested reader a standard verification that $f$ is not $\mathrm{x}$-continuous at $(0,0,0)$.

3. ONE-PROMISING HYPOTHESIS.

Observe that the definition of $x$-continuity at $(p, q, r)$ requires the existence of a "sma11" neighborhood $U$ ' of $p$ and "small" nonempty open sets $V^{\prime}$ and $W^{\prime}$ such that $q$ and $r$ "clusters" to $V^{\prime}$ and $W^{\prime}$ respectively and such that the set $f\left(U^{\prime} \times V^{\prime} \times W^{\prime}\right)$ is contained in a "small", previously chosen, open set $\mathrm{N}$. This observation prompts us to label this kind of product almost continuity as 1-3-continuity - since we require the existence of only one "sma11" neighborhood $U$ ' (around p) of the three neighborhoods $\mathrm{U}, \mathrm{V}, \mathrm{W}$.

The term "1-3-continuity" has been used already, in a different sense in Breckenridge and Nishiura [9].

So, now let us consider "2-3-continuity".

More precisely, given spaces $X, Y, Z$ and $T$, we say that $f: X \times Y \times Z \rightarrow T$ is 2-3-continuous or more specifically $x y$-continuous, if for every $(\mathrm{p}, \mathrm{q}, \mathrm{r}) \varepsilon \mathrm{X} \times \mathrm{Y} \times \mathrm{Z}$, for every neighborhood $U \times V \times W$ of $(p, q, r)$ and for every neighborhood $N$ of $f(p, q, r)$ there is a neighborhood $U^{\prime}$ of $p$, with $U^{\prime} \subset U$, there is a neighborhood $v^{1}$ of $q$, with $V^{1} \subset V$ and a nonempty open set $W^{1}$, with $W^{1} \subset W$ such that for all $(\mathrm{x}, \mathrm{y}, \mathrm{z}) \in \mathrm{U}^{1} \times \mathrm{v}^{1} \times \mathrm{W}^{1}$ we have $\mathrm{f}(\mathrm{x}, \mathrm{y}, \mathrm{z}) \varepsilon \mathrm{N}$.

Now, 3-3-continuity can be defined easily; the set $W^{1}$ in definition of 2-3-continuity is assumed to be a neighborhood of $r$ - not just only a nonempty open subset of $W$. 
Clearly, every 3-3-continuous ( $\equiv$ continuous) function is 2-3-continuous;

2-3-continuous functions are 1-3-continuous and the latter are in turn 0-3-continuous (三 quasi-continuous).

It now follows from a result of $T$. Neubrunn [10] that if $X, Y, Z$ are "nice" (e.g. Baire, second countable), T-regular then if $\mathrm{f}: \mathrm{X} \times \mathrm{Y} \times \mathrm{Z} \rightarrow \mathrm{T}$ is separately quasi-continuous then it is (jointly) quasi-continuous.

We can present this fact in the following symbolic equality:

$$
" 0+0+0=0 ",
$$

where the numbers ( 0 or 1 ) on the left side of the equality stand for quasi-continuity (0) or continuity (1) of the corresponding sections and the numbers on the right $(i=0,1,2$ or 3 ) denote the corresponding i-3-continuity of $f$ as a function of three variables.

Theorem $A$ implies that if $X, Y, Z$ and $T$ are as above and if $f: X \times Y \times Z \rightarrow T$ is continuous in $x$ and is quasi-continuous in $y$ and is quasi-continuous in $z$, then $f$ is 1-3-continuous. Consequently, we get:

$$
" 1+0+0=1 " \text {. }
$$

In view of the above considerations it is now natural to state the following: HYPOTHESIS. Let $X, Y$ and $Z$ be Baire, second countable spaces and let $T$ be regular. If $\mathrm{f}: \mathrm{X} \times \mathrm{Y} \rightarrow \mathrm{Z}$ T is:

1) continuous in $x$, and

2) continuous in $y$, and

3) quasi-continuous in $z$,

Then $f$ is 2-3-continuous;

In other words:

$$
" 1+1+0=2 "
$$

We shall resolve this Hypothesis in the negative in the forthcoming Example 8 . Now we shall exhibit two examples of 1-3-continuous functions which are not $(i+1)-3$-continuous, $i=1,2$.

EXAMPLE 8. A 1-3-continuous functin which is not 2-3-continuous. Let f: $\mathbb{R}^{3} \rightarrow \mathbb{R}$ be given by $f\left(x_{1}, x_{2}, x_{3}\right)=g\left(x_{1}, x_{2}\right)$ where $g$ is an arbitrary separately continuous function which is discontinuous at $(0,0)$.

EXAMPLE 9. A 2-3-continuous function which is not 3-3-continuous (三 continuous). Take $\mathrm{f}: \mathbb{R}^{3} \rightarrow \mathbb{R}$ to be $\mathrm{f}\left(\mathrm{x}_{1}, \mathrm{x}_{2}, \mathrm{x}_{3}\right)=\mathrm{h}\left(\mathrm{x}_{3}\right)$, where $\mathrm{h}$ is any function which is continuous except for 0 .

Using the above pattern the reader will easily construct 0-3-continuous function (三 quasi-continuous) which is not 1-3-continuous.

Apparently, the above constructions can be illustrated with the following very specific formula-ready example.

EXAMPLE 10. Let $\mathrm{f}: \mathbb{R}^{3} \rightarrow \mathbb{R}$ be a function. 


$$
\begin{aligned}
& f\left(x_{1}, x_{2}, x_{3}\right)=g_{i}^{3}\left(x_{1}, \ldots, x_{i}\right), i=1,2 \text { where }
\end{aligned}
$$

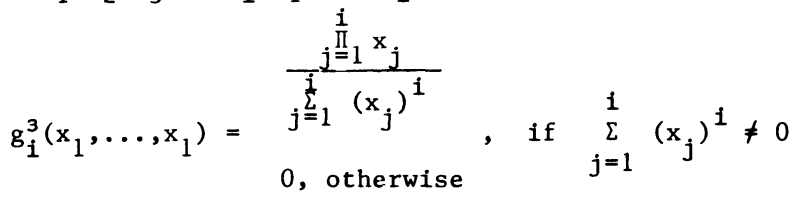

Then $f$ is $i-3$-continuous which is not $(i+1)-3$-continuous, $1=1,2$.

4. FURTHER GENERALIZATION OF i-3-CONTINUITY.

Having defined $1-3$ and 2-3-continuity for $f: x_{1} \times x_{2} \times x_{3} \rightarrow T$, we shall now extend these ideas to a general case.

Namely, let $\mathrm{n}$ be an arbitrary natural number. We say that $\mathrm{f}$ function

$\mathrm{f}: \prod_{i=1}^{n} \mathrm{x}_{i} \quad \mathrm{~T}$ is A-n-continuous if for every $\left(\mathrm{p}_{1}, \mathrm{p}_{2}, \ldots, \mathrm{p}_{\mathrm{n}}\right) \prod_{i=1}^{n} \mathrm{x}_{1}$ and for every neighborhood $U_{1} \times U_{2} \times \ldots \times U_{n}$ of $\left(p_{1}, p_{2}, \ldots, p_{n}\right)$ and for every neighborhood $N$ of $f\left(p_{1}, p_{2}, \ldots, p_{n}\right)$ there are neighborhoods $U_{i, s}^{\prime}(1 \leqq s \leqq k)$ of the first $k$ out of $n$ points $p_{1}, p_{2}, \ldots, p_{n}$ with $U_{i, s}^{\prime} \subset U_{i}$ and there are $(n-k)$ nonempty open sets $V_{k}^{\prime} V_{i, m}^{\prime}$ with $V_{i, m}^{\prime} \subset U_{i} 1 \leqq m \leqq n-k$ such that for all $\left(x_{1}, x_{2}, \ldots, x_{n}\right) \varepsilon \prod_{s=1}^{k} U_{1, s}^{\prime} \times \prod_{m=1}^{n-k} V_{1, m}^{1, m}$ we have $f\left(x_{1}, x_{2}, \ldots, x_{n}\right) \in N$.

An interested reader will easily observe that the formula

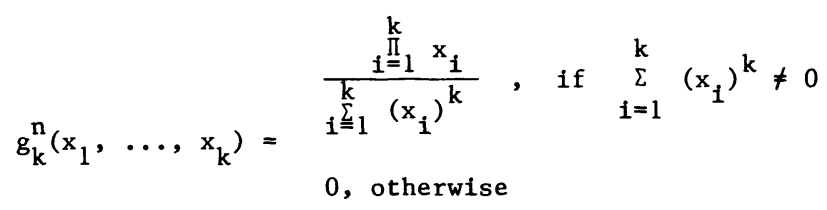

where $f: \mathbb{R}^{n} \rightarrow \mathbb{R}$ describes a $k-n$-continuous function $f$ given by $f\left(x_{1}, \ldots, x_{n}\right)=g_{k}^{n}\left(x_{1}, \ldots, x_{k}\right), k=1,2,3, \ldots n-1$.

One can also give analogues of Example 8 and 9 for $k$-n-continuity.

Studies of $\mathrm{C}(\mathrm{f})$ in hyperspaces for separately continuous functions and related ones were done also in Bögel [11] and Hahn [12].

5. A PARTIAL SOLUTiON TO A PROBLEM OF M. TALAGRAND.

M. Talagrand ([13] Problem 3 p. 160) asked whether if $X$ is Baire, $Y$ is compact and $f: X \times Y \rightarrow \mathbb{R}$ is any separately continuous function, is there the set $C(f)$ of points of continuity of $f$ nonempty.

We shall answer this question in the positive if a compact space $\mathrm{Y}$ is additionally first countable.

In fact, we have shown the following result:

LEMMA 11. (Lee and Plotrowski [2], Lemma 2 p. 381). Let X be Baire, $Y$ be first countable and $\mathrm{Z}$ be regular. If $\mathrm{f}: \mathrm{X} \times \mathrm{Y} \rightarrow \mathrm{Z}$ is a function such that all its $x$-sections $f_{X}$ are continuous with the exception of a first category set, and all its $y$-sections $f_{Y}$ are quasi-continuous, then $f$ is quasi-continuous with respect to $y$.

It follows from the definition that

REMARK 12. Every quasi-continuous function with respect to $y$ is quasi-continuous.

LEMMA 13. (Marcus [14]). Let $X$ be a Baire, $M$ be metric. If $f: X \rightarrow M$ is quasicontinuous, then $C(f)$, the set of point of continuity of $f$ is dense $G_{\delta}$ subset of $X$. 
PROPOSITION 14. Let $X$ be Baire, $Y$ be compact first countable and let

$f: X \quad Y \rightarrow \mathbb{R}$ be any separately continuous function. Then $C(f) \neq 0$.

PROJF. By Lemma 11 and Remark 12 such $\mathrm{f}$ is quasi-continuous. Now, since the Cartesian product of a compact space and a Baire space is Baire, we are done by Lemma 13.

ACKNOWLEDGEMENT: The second author would like to express his thanks to the Research Council of Youngstown State University for a grant which enables us to complete this research.

\section{REFERENCES}

1. KEMPISTY, S. Sur les fonctions quasicontinues, Fund. Math. 19 (1932), $183-197$.

2. LEE, J.P. and PIOTROWSKI, Z. On Kempisty's generalized continuity, Rend. Circ. Matem. Palermo XXXIV (1985), 380-386.

3. ARHANGEL'SKII, A. On topological spaces which are complete in the sense of Cech (Russian), Vest. Mosc. Univ., Ser. Math 2 (1961), 37-40.

4. FROLIK, Z. Generalization of the G property of complete metric spaces, Czech. Math. J. 10 (1960), 359-379.

5. ENGELKING, R. General Topology PWN - Polish Scientific Publishers, Warszawa, 1977.

6. ОХтовY, J.C. Cartesian products of Baire spaces, Fund. Math. 49 (1961), 154-166.

7. FROLIK, Z. Baire spaces and some generalizations of complete metric spaces, Czech. Math. J. 11 (1961), 237-248.

8. MARTIN, N.F.G. Quasi-continuous functions on product spaces, Duke Math. J. 28 (1961), 39-44.

9. BRECKENRIDGE, J.C. and NISHIURA, T. Partial continuity, quasi-continuity, and Baire spaces, Bul1. Inst. Math. Acad. Sinica 4 (1976), 191-203.

10. NEUBRUNN, T. A generalized continuity and product spaces, Math. Slovaca 26 (1976) 97-99.

11. BOGEL, K. Uher die stetigkeit und die Schwankung von funktionen zweiler reeller Veranderlicher, Math. Ann. 81 (1920), 64-93.

12. HAHN, H. Reelle functionen, Leipzig, 1932.

13. TALAGRAND, M. Espaces de Baire et espaces de Namioka, Math. Ann. 270 (1985), 159-164.

14. MARCUS, S. Sur les fonctione quasi-continues au sens de S. Kempisty, Collog. Math. 8 (1961), 47-53. 


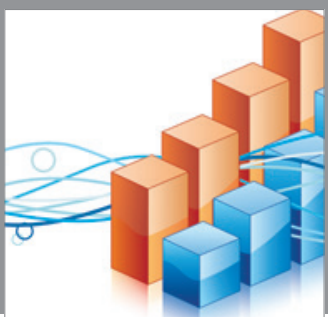

Advances in

Operations Research

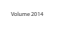

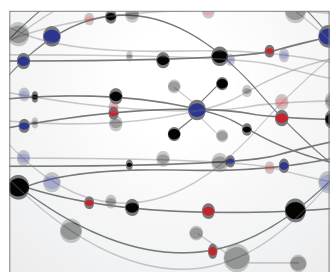

\section{The Scientific} World Journal
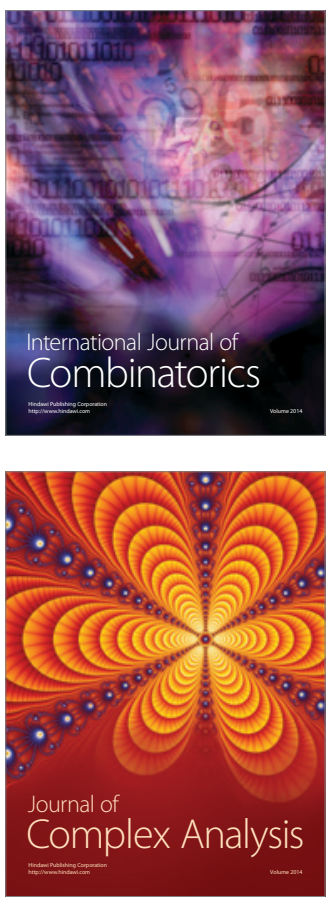

International Journal of

Mathematics and

Mathematical

Sciences
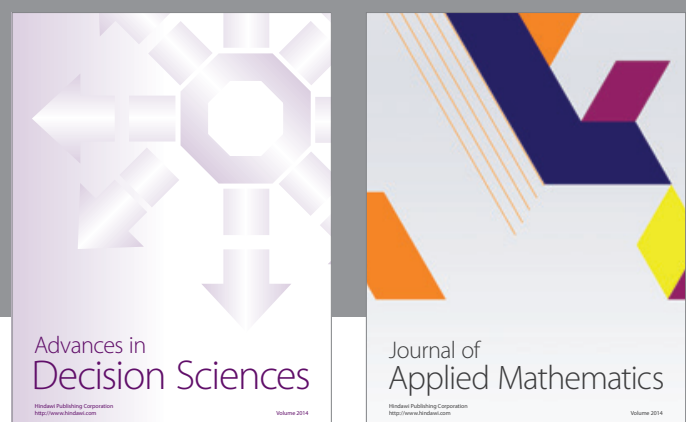

Journal of

Applied Mathematics
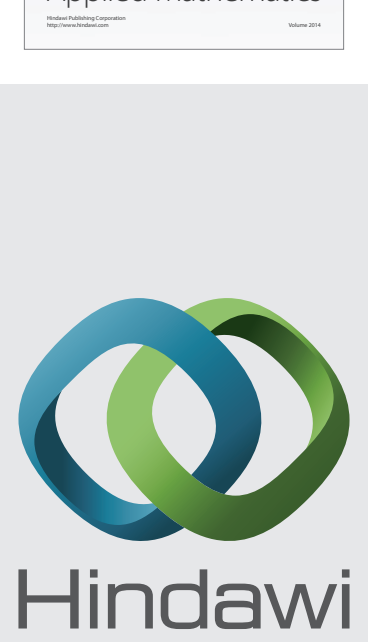

Submit your manuscripts at http://www.hindawi.com
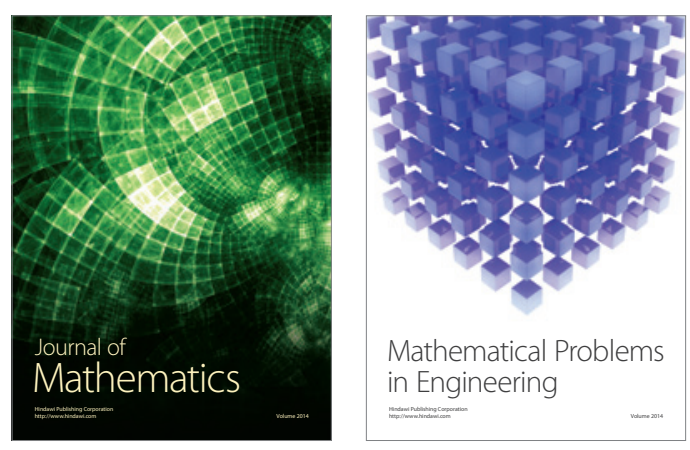

Mathematical Problems in Engineering
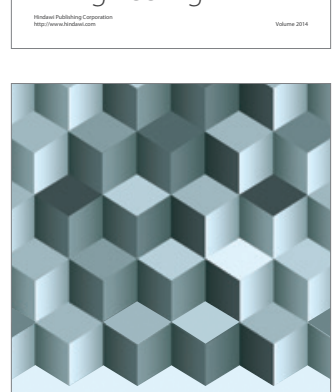

Journal of

Function Spaces
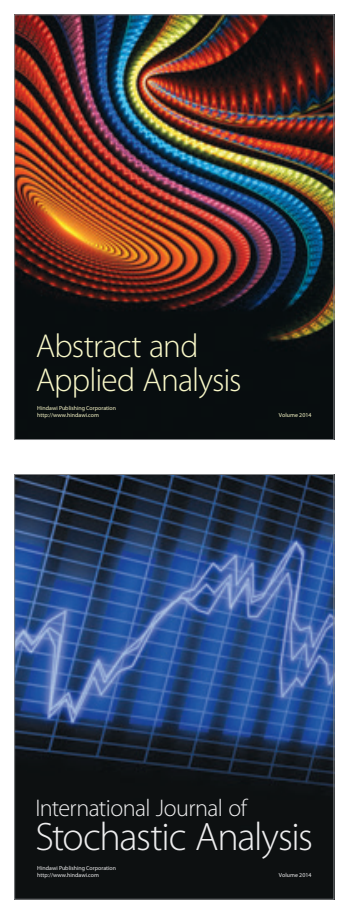

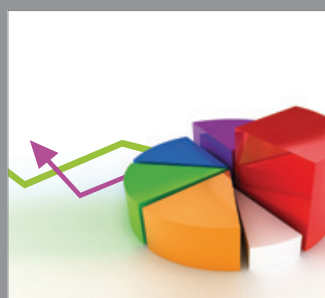

ournal of

Probability and Statistics

Promensencen
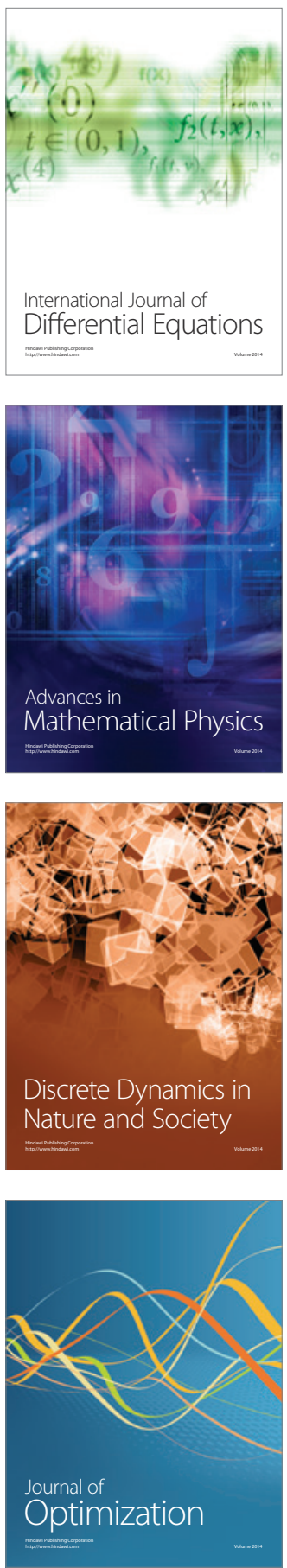